\title{
The meta-abelian elliptic KZB associator and periods of Eisenstein series
}

\author{
Nils Matthes ${ }^{1}$
}

Published online: 21 November 2017

(C) The Author(s) 2017. This article is an open access publication

\begin{abstract}
We compute the image of Enriquez' elliptic KZB associator in the (maximal) meta-abelian quotient of the fundamental Lie algebra of a once-punctured elliptic curve. Our main result is an explicit formula for this image in terms of Eichler integrals of Eisenstein series, and is analogous to Deligne's computation of the depth one quotient of the Drinfeld associator. We also show how to retrieve Zagier's extended period polynomials of Eisenstein series, as well as the values at zero of Beilinson-Levin's elliptic polylogarithms from the meta-abelian elliptic KZB associator.
\end{abstract}

Keywords Modular symbols · Elliptic associators · Elliptic polylogarithms

\section{Mathematics Subject Classification 11F67}

\section{Introduction}

This paper deals with the computation of some of the coefficients of the elliptic KZB associator defined by Enriquez [14]. In order to put things into context, we first recall the analogous picture in genus zero, due to Deligne, Drinfeld and Ihara.

Let $\mathfrak{p}(U):=\mathbb{L}\left(\mathbf{x}_{0}, \mathbf{x}_{1}\right)^{\wedge}$ be the lower central series completion of the free Lie algebra in variables $x_{0}, x_{1}$, and denote by $\exp \mathfrak{p}(U)$ the associated pro-unipotent algebraic group. The Drinfeld associator $\Phi\left(\mathbf{x}_{0}, \mathbf{x}_{1}\right)$ is an element of $\exp \mathfrak{p}(U)_{\mathbb{R}}:=$ $\exp (\mathfrak{p}(U) \widehat{\otimes} \mathbb{R})$, which is constructed from the monodromy of the universal KnizhnikZamolodchikov (KZ) connection on $\mathbb{P}_{\mathbb{C}}^{1} \backslash\{0,1, \infty\}$ (for this reason, $\Phi$ is sometimes

Nils Matthes

nilsmath@mpim-bonn.mpg.de

1 Max-Planck-Institut für Mathematik, Vivatsgasse 7, 53111 Bonn, Germany

Birkhäuser 
called KZ-associator). First introduced in [13], the Drinfeld associator plays a pivotal role in the context of quantum groups and Grothendieck-Teichmüller theory.

We are interested in arithmetic properties of $\Phi\left(\mathrm{x}_{0}, \mathrm{x}_{1}\right)$. The following two aspects, which are in fact closely related to each other, are of particular relevance.

(i) The coefficients of $\Phi\left(\mathbf{x}_{0}, \mathbf{x}_{1}\right)$ are expressible as $\mathbb{Q}$-linear combinations of multiple zeta values

$$
\zeta\left(k_{1}, \ldots, k_{n}\right)=\sum_{m_{1}>\cdots>m_{n}>0} \frac{1}{m_{1}^{k_{1}} \cdots m_{n}^{k_{n}}}, \quad k_{1} \geq 2, k_{2}, \ldots, k_{n} \geq 1,
$$

which are generalizations of the special values of the Riemann zeta function at positive integers. These numbers have (at least conjecturally) a rich algebraic structure $[16,20]$.

(ii) The Lie algebra $\mathfrak{p}(U)$ is the de Rham realization of an element of the category MTM of mixed Tate motives over $\mathbb{Z}$ ([12], §5). As a consequence, the unipotent fundamental group $\mathcal{U}_{\text {MTM }}$ of MTM acts on $\exp \mathfrak{p}(U)$ (Ihara action), and in particular on $\Phi\left(\mathbf{x}_{0}, \mathbf{x}_{1}\right){ }^{1}$ The Deligne-Ihara conjecture (proved by Brown in [3]) states that this action is faithful, thus elements of $\mathcal{U}_{\text {MTM }}$ are completely determined by their action on $\Phi\left(\mathbf{x}_{0}, \mathbf{x}_{1}\right)$, which can be computed very explicitly [4].

For both (i) and (ii), the archetypal result is due to Deligne ([11], §19), who inspired by unpublished work of Wojtkowiak essentially showed that

$$
\log \left(\Phi\left(\mathbf{x}_{0}, \mathbf{x}_{1}\right)\right) \equiv-\sum_{k=2}^{\infty} \zeta(k) \operatorname{ad}^{k-1}\left(\mathbf{x}_{0}\right)\left(\mathbf{x}_{1}\right) \quad \bmod \left[D^{1} \mathfrak{p}(U), D^{1} \mathfrak{p}(U)\right]
$$

where $D^{1} \mathfrak{p}(U) \subset \mathfrak{p}(U)$ denotes the ideal generated by $\mathrm{x}_{1}$. On the one hand, this exhibits the Riemann zeta values $\zeta(k)$ as coefficients of $\log \left(\Phi\left(\mathbf{x}_{0}, \mathbf{x}_{1}\right)\right)$. On the other hand, since $\zeta(k) \neq 0$, one deduces from (1.1) that the generators $\exp \left(\sigma_{2 n+1}\right)$ of $\mathcal{U}_{\text {MTM }}$ act non-trivially on $\exp \mathfrak{p}(U)([12], \S 6.8)$, which was a first step towards establishing the Deligne-Ihara conjecture.

In this paper, we consider an elliptic analog of the above situation. Let $\mathfrak{H}$ be the Poincaré upper half-plane, and consider for $\tau \in \mathfrak{H}$ the once-punctured, complex elliptic curve $E_{\tau}^{\times}:=\mathbb{C} /(\mathbb{Z}+\mathbb{Z} \tau) \backslash\{0\}$. Following Hain-Matsumoto [19], we denote its de Rham fundamental group by $\mathfrak{p}\left(E_{\tau}^{\times}\right) \cong \mathbb{L}(\mathrm{a}, \mathrm{b})^{\wedge}$. In [14], Enriquez constructs the elliptic KZB associator $(A(\tau), B(\tau)) \in \exp \mathfrak{p}\left(E_{\tau}^{\times}\right) \mathbb{C} \times \exp \mathfrak{p}\left(E_{\tau}^{\times}\right) \mathbb{C}$ from the monodromy of the universal elliptic Knizhnik-Zamolodchikov-Bernard (KZB) connection [9,23]. It is an elliptic version of the Drinfeld associator, and the analogs of (i) and (ii) above are the following.

(i) The coefficients of the elliptic KZB associator are the elliptic multiple zeta values, first introduced in [15] and studied in more detail in [2, 24,26,27]. They are closely related to both multiple zeta values and to iterated integrals of Eisenstein series $[6,25]$.

\footnotetext{
1 In this context, $\Phi\left(\mathrm{x}_{0}, \mathrm{x}_{1}\right)$ is usually denoted $d c h$ (for 'droit chemin').
} 
(ii) The Lie algebra $\mathfrak{p}\left(E_{\tau}^{\times}\right)$, viewed as a local system over the moduli space $\mathcal{M}_{1, \overrightarrow{1}}$ of elliptic curves with a non-zero tangent vector at the origin, is the de Rham realization of an element of the category $\mathrm{MEM}_{\overrightarrow{1}}$ of universal mixed elliptic motives (over $\mathcal{M}_{1, \overrightarrow{1}}$ ). This category can be seen as an elliptic enhancement of the category of mixed Tate motives over $\mathbb{Z}$. The corresponding Galois group $\mathcal{G}_{\mathrm{MEM}_{1}}$ acts on $\mathfrak{p}\left(E_{\tau}^{\times}\right)$[19], and therefore also on the elliptic KZB associator. In analogy to the Deligne-Ihara conjecture, it is asked in [19], §24.2 whether the action of $\mathcal{G}_{\mathrm{MEM}_{\overrightarrow{1}}}$ on $\mathfrak{p}\left(E_{\tau}^{\times}\right)$is faithful.

The main goal of this article is to establish an analog of (1.1) for the elliptic KZB associator, i.e. the explicit computation of the images of the formal logarithms $\mathfrak{A}(\tau):=$ $\log (A(\tau))$ and $\mathfrak{B}(\tau):=\log (B(\tau))$ in a certain quotient of $\mathfrak{p}\left(E_{\tau}^{\times}\right) \mathbb{C}$. More precisely, let $D^{1} \mathfrak{p}\left(E_{\tau}^{\times}\right) \subset \mathfrak{p}\left(E_{\tau}^{\times}\right) \cong \mathbb{L}(\mathrm{a}, \mathrm{b})^{\wedge}$ be the commutator. Taking its lower central series defines a filtration $D^{\bullet} \mathfrak{p}\left(E_{\tau}^{\times}\right)$, the elliptic depth filtration ([19], §27). In particular, $D^{2} \mathfrak{p}\left(E_{\tau}^{\times}\right)$is the double commutator, and our goal is to compute the images $\mathfrak{A}(\tau)^{\text {met-ab }}$ and $\mathfrak{B}(\tau)^{\text {met }-\mathrm{ab}}$ of the elliptic KZB associator in the meta-abelian quotient

$$
\mathfrak{p}\left(E_{\tau}^{\times}\right)_{\mathbb{C}}^{\text {met-ab }}:=\mathfrak{p}\left(E_{\tau}^{\times}\right)_{\mathbb{C}} / D^{2} \mathfrak{p}\left(E_{\tau}^{\times}\right)_{\mathbb{C}} \cong(\mathbb{C} \cdot \mathrm{a} \oplus \mathbb{C} \cdot \mathrm{b}) \oplus \mathbb{C} \llbracket U, V \rrbracket,
$$

where $U^{k} V^{l}:=\operatorname{ad}^{k}(\mathrm{a}) \operatorname{ad}^{l}(\mathrm{~b})([\mathrm{a}, \mathrm{b}])$. Our main result can then be stated as follows.

Theorem (Theorem 5.6 below) Let $\overline{\mathrm{U}}:=\frac{\mathrm{U}}{2 \pi i}$ and $\mathrm{W}:=\overline{\mathrm{U}}+\tau \mathrm{V}$. We have

$$
\mathfrak{A}(\tau)^{\mathrm{met}-\mathrm{ab}}=2 \pi i \mathrm{~b}+\exp \left(\tau \frac{\partial}{\partial \overline{\mathrm{U}}} \mathrm{V}\right) \mathfrak{A}_{\infty}^{(1)}-2 \pi i \mathrm{~V} \sum_{k=1}^{\infty} \frac{2}{(2 k-2) !} \int_{\tau}^{\overrightarrow{1}_{\infty}} \underline{G}_{2 k}
$$

and

$$
\mathfrak{B}(\tau)^{\mathrm{met}-\mathrm{ab}}=\mathrm{a}+2 \pi i \tau \mathrm{b}+\exp \left(\tau \frac{\partial}{\partial \overline{\mathrm{U}}} \mathrm{V}\right) \mathfrak{B}_{\infty}^{(1)}-2 \pi i \mathrm{~W} \sum_{k=1}^{\infty} \frac{2}{(2 k-2) !} \int_{\tau}^{\overrightarrow{1}_{\infty}} \underline{G}_{2 k}
$$

Here, $\int_{\tau}^{\overrightarrow{1}_{\infty}} \underline{G}_{2 k}:=(2 \pi i)^{2 k-1} \int_{\tau}^{\overrightarrow{1}_{\infty}} G_{2 k}(z)(\mathrm{W}-z \mathrm{~V})^{2 k-2} \mathrm{~d} z$ is the regularized Eichler integral of $G_{2 k}([6], \S 4)$, and the series $\mathfrak{A}_{\infty}^{(1)}, \mathfrak{B}_{\infty}^{(1)}$ are given by

$$
\begin{aligned}
& \mathfrak{A}_{\infty}^{(1)}=2 \pi i\left(c(\mathrm{U})-\frac{(2 \pi i)}{4} \mathrm{~V}+\sum_{n \geq 3, \text { odd }} \zeta(n) \mathrm{V}^{n}\right) \\
& \mathfrak{B}_{\infty}^{(1)}=-2 \pi i(c(2 \pi i \mathrm{~V})-\mathrm{U} c(\mathrm{U}) c(2 \pi i \mathrm{~V}))+\sum_{n \geq 3, \text { odd }} \zeta(n) \mathrm{UV}^{n-1}
\end{aligned}
$$

where $c(x)=\frac{1}{e^{x}-1}+\frac{1}{2}-\frac{1}{x}=\sum_{k=2}^{\infty} \frac{B_{k}}{k !} x^{k-1}$.

Similar considerations have been made by Hain to prove that the generators $\exp \left(\mathbf{e}_{2 k}\right)$ of the geometric fundamental group $\mathcal{G}_{\mathrm{MEM}}^{\text {geom }}$ act non-trivially on $\mathfrak{p}\left(E_{\tau}^{\times}\right)$([18], Theorem 15.7). Moreover, our theorem gives a closed expression of elliptic multiple zeta 
values of depth one explicitly in terms of Riemann zeta values and Eichler integrals of Eisenstein series.

The proof of Theorem 5.6 uses a result of Enriquez [14] to the effect that

$$
\mathfrak{A}(\tau)=g(\tau)\left(\mathfrak{A}_{\infty}\right), \quad \mathfrak{B}(\tau)=g(\tau)\left(\mathfrak{B}_{\infty}\right),
$$

for certain explicit elements $\mathfrak{A}_{\infty}, \mathfrak{B}_{\infty} \in \mathfrak{p}\left(E_{\tau}^{\times}\right) \mathbb{C}$ and an automorphism $g(\tau) \in$ $\operatorname{Aut}\left(\exp \left(\mathfrak{p}\left(E_{\tau}^{\times}\right)_{\mathbb{C}}\right)\right)$. Then, we separately compute the images of $\mathfrak{A}_{\infty}$ and $\mathfrak{B}_{\infty}$ in $\mathfrak{p}\left(E_{\tau}^{\times}\right)_{\mathbb{C}}^{\text {met }-\mathrm{ab}}$ and of $g(\tau)$ in $\operatorname{Aut}\left(\exp \left(\mathfrak{p}\left(E_{\tau}^{\times}\right)_{\mathbb{C}}^{\text {met-ab }}\right)\right)$, and from this, we are able to deduce Theorem 5.6.

The series $\mathfrak{A}_{\infty}$ and $\mathfrak{B}_{\infty}$ are arithmetic: they can be expressed in terms of the Drinfeld associator and therefore come from genus zero. On the other hand, the automorphism $g(\tau)$ is geometric: it describes the action of $\mathcal{G}_{\mathrm{MEM}}^{\text {geom }}$ on $\exp \mathfrak{p}\left(E_{\tau}^{\times}\right)$. As a byproduct of our proof, we see that already their images in the meta-abelian quotient are interesting objects in their own right. Namely, the automorphism $g(\tau)^{\text {met-ab }}$ is essentially the generating series of the special values of elliptic polylogarithms at the zero section of the elliptic curve [1,22] (cf. Theorem 5.4 and Corollary 5.5), while $\mathfrak{A}_{\infty}^{\text {met-ab }}, \mathfrak{B}_{\infty}^{\text {met-ab }}$ turn out to be generating series of the extended period polynomials of Eisenstein series [33] (cf. Theorem 5.2 and Corollary 5.3).

Finally, we note that Nakamura [28,29] has studied an $\ell$-adic analog of the metaabelian image of the elliptic KZB associator (called "universal power series for Dedekind sums"), which is a genus one analog of Ihara's universal power series for Jacobi sums [21]. It would be very interesting to compare his results to ours.

The plan of the paper is as follows. In Sects. 2 and 3, we collect some background in order to make the paper self-contained. Then, in Sect. 4, we recall the definition of the elliptic KZB associator [14], but from the point of view of the mixed Hodge structure on the unipotent fundamental group of $E_{\tau}^{\times}$[8]. Finally, in Sect. 5, the main results of this paper are proved.

\section{Preliminaries}

\subsection{Notation and conventions}

We start by introducing some general notation, to be used throughout the text.

We denote by $\mathfrak{H}:=\{z \in \mathbb{C} \mid \operatorname{Im}(z)>0\}$ the upper half-plane, with canonical coordinate $\tau$. For $\tau \in \mathfrak{H}$, we let $E_{\tau}^{\times}:=\mathbb{C} /(\mathbb{Z}+\mathbb{Z} \tau) \backslash\{0\}$ be the associated oncepunctured complex elliptic curve.

For any finite set $\left\{\mathbf{x}_{1}, \ldots, \mathbf{x}_{n}\right\}$ and a field $K$, we denote by $\mathbb{L}\left(\mathbf{x}_{1}, \ldots, \mathbf{x}_{n}\right)_{K}$ the free Lie algebra on $X$ over $K$ (we omit $K$ if $K=\mathbb{Q}$ ), and by $\mathbb{L}\left(\mathrm{x}_{1}, \ldots, \mathrm{x}_{n}\right)_{K}^{\wedge}$ the completion for its lower central series. It is a topological Lie algebra over $K$, whose topology is induced from the lower central series. Its topological universal enveloping algebra is given by $K\left\langle\left\langle\mathrm{x}_{1}, \ldots, \mathrm{x}_{n}\right\rangle\right\rangle$, the $K$-algebra of formal power series in the noncommuting variables $\mathbf{x}_{1}, \ldots, \mathbf{x}_{n}$, and the exponential map exp : $\mathbb{L}\left(\mathbf{x}_{1}, \ldots, \mathbf{x}_{n}\right)_{K}^{\wedge} \rightarrow$ $K\left\langle\left\langle\mathrm{x}_{1}, \ldots, \mathrm{x}_{n}\right\rangle\right\rangle$ defines an isomorphism onto the subspace of $K\left\langle\left\langle\mathrm{x}_{1}, \ldots, \mathrm{x}_{n}\right\rangle\right\rangle$ of group- 
like elements, denoted by $\exp \mathbb{L}\left(\mathbf{x}_{1}, \ldots, \mathbf{x}_{n}\right)_{K}^{\wedge}$. For more background, we refer to [30,31].

\subsection{Derivations on the fundamental Lie algebra of a once-punctured elliptic curve}

Following [19], we will denote by $\mathfrak{p}\left(E_{\tau}^{\times}\right)$the (de Rham) fundamental Lie algebra of the once-punctured elliptic curve $E_{\tau}^{\times}$. With notation as above, one has

$$
\mathfrak{p}\left(E_{\tau}^{\times}\right) \cong \mathbb{L}(\mathrm{a}, \mathrm{b})^{\wedge}
$$

where the generators $\mathrm{a}, \mathrm{b}$ correspond to the natural homology cycles on $E_{\tau}^{\times}$.

We will need to consider a special family of derivations on $\mathfrak{p}\left(E_{\tau}^{\times}\right)$. Denote by $\operatorname{Der}^{0}\left(\mathfrak{p}\left(E_{\tau}^{\times}\right)\right)$the Lie algebra of continuous derivations $D$, which satisfy $D([\mathrm{a}, \mathrm{b}])=0$ and such that $D(\mathrm{~b})$ has no linear term in a. From these two conditions, it follows easily that every $D \in \operatorname{Der}^{0}\left(\mathfrak{p}\left(E_{\tau}^{\times}\right)\right)$is uniquely determined by its value on a.

Definition 2.1 (Tsunogai) For every $k \geq 0$, define $\varepsilon_{2 k} \in \operatorname{Der}^{0}\left(\mathfrak{p}\left(E_{\tau}^{\times}\right)\right)$by its value on a:

$$
\varepsilon_{2 k}(\mathrm{a})= \begin{cases}-\mathrm{b} & k=0 \\ \frac{2}{(2 k-2) !} \mathrm{ad}^{2 k}(\mathrm{a})(\mathrm{b}) & k>0\end{cases}
$$

We also let $\mathfrak{u} \subset \operatorname{Der}^{0}\left(\mathfrak{p}\left(E_{\tau}^{\times}\right)\right)$be the Lie subalgebra generated by the $\varepsilon_{2 k}$.

The derivations $\varepsilon_{2 k}$ have first been introduced by Tsunogai ([32], §3) in the context of Galois actions on fundamental groups of punctured elliptic curves. They also play an important role in the theory of universal mixed elliptic motives, as the relative unipotent completion of $\mathrm{SL}_{2}(\mathbb{Z})$ acts on $\mathfrak{p}\left(E_{\tau}^{\times}\right)$through them ([19], §20).

Remark 2.2 The value of $\varepsilon_{2 k}$ on $\mathrm{b}$ is given by

$$
\varepsilon_{2 k}(\mathrm{~b})=\frac{2}{(2 k-2) !} \sum_{0 \leq j<k}(-1)^{j}\left[\operatorname{ad}^{j}(\mathrm{a})(\mathrm{b}), \mathrm{ad}^{2 k-1-j}(\mathrm{a})(\mathrm{b})\right] \text {. }
$$

In particular, $\varepsilon_{0}(\mathrm{~b})=0$.

\subsection{Eichler integrals of Eisenstein series}

Consider the Hecke-normalized Eisenstein series for $\mathrm{SL}_{2}(\mathbb{Z})$ of weight $2 k$ :

$$
G_{2 k}(q):= \begin{cases}-\frac{B_{2 k}}{4 k}+\sum_{n=1}^{\infty}\left(\sum_{d \mid n} d^{2 k-1}\right) q^{n} & k \geq 1 \\ -1 & k=0\end{cases}
$$

where $B_{2 k}$ denotes the $2 k$-th Bernoulli number and $q=e^{2 \pi i \tau}$. Extending earlier work of Manin [25], Brown [6] introduced (regularized) iterated integrals of (2.1) (or iterated Eisenstein integrals for short) 


$$
\mathcal{G}\left(2 k_{1}, \ldots, 2 k_{n} ; \tau\right):=\int_{\tau}^{\overrightarrow{1}_{\infty}} G_{2 k_{1}}\left(\tau_{1}\right) \ldots G_{2 k_{n}}\left(\tau_{n}\right) \mathrm{d} \tau_{1} \ldots \mathrm{d} \tau_{n}
$$

where $\overrightarrow{1}_{\infty}$ denotes the tangential base point 1 at $i \infty$. We refer to [6], $\$ 4$, for the general definition, and only note the special case

$$
\begin{aligned}
\mathcal{G}\left(\{0\}_{n}, 2 k ; \tau\right) & =(-1)^{n} \int_{\tau \leq \tau_{1} \leq \ldots \tau_{n+1} \leq i \infty} \ldots \int_{2 k}\left(\tau_{n+1}\right)-a_{0}\left(G_{2 k}\right) \mathrm{d} \tau_{1} \ldots \mathrm{d} \tau_{n+1} \\
& -a_{0}\left(G_{2 k}\right) \frac{\tau^{n+1}}{(n+1) !}
\end{aligned}
$$

where $\{0\}_{n}$ denotes an $n$-tuple of zeros, and $a_{0}\left(G_{2 k}\right)=-\frac{B_{2 k}}{4 k}$ is the constant term in the Fourier expansion (2.1) of $G_{2 k}$. From the shuffle product formula for (regularized) iterated integrals ([6], Proposition 4.7), we further deduce

$$
\mathcal{G}\left(\{0\}_{n-1}, 2 k, 0 ; \tau\right)=\mathcal{G}(0 ; \tau) \mathcal{G}\left(\{0\}_{n-1}, 2 k ; \tau\right)-n \mathcal{G}\left(\{0\}_{n}, 2 k ; \tau\right)
$$

Both $\mathcal{G}\left(\{0\}_{n}, 2 k ; \tau\right)$ and $\mathcal{G}\left(\{0\}_{n-1}, 2 k, 0 ; \tau\right)$ can be expressed in terms of generalized Eichler integrals

$$
I_{n}\left(G_{2 k} ; \tau\right):=\int_{\tau}^{i \infty}\left[G_{2 k}(z)-a_{0}\left(G_{2 k}\right)\right](\tau-z)^{n} \mathrm{~d} z-\int_{0}^{\tau} a_{0}\left(G_{2 k}\right)(\tau-z)^{n} \mathrm{~d} z
$$

with the classical Eichler integral of $G_{2 k}$ being the special case $n=2 k-2$ and $k \geq 2$ (cf. e.g. [34], §1).

Proposition 2.3 We have

$$
\begin{aligned}
\mathcal{G}\left(\{0\}_{n} ; \tau\right) & =\frac{\tau^{n}}{n !} \\
\mathcal{G}\left(\{0\}_{n}, 2 k ; \tau\right) & =\frac{1}{n !} I_{n}\left(G_{2 k} ; \tau\right),
\end{aligned}
$$

and for $k, n \geq 1$ :

$$
\mathcal{G}\left(\{0\}_{n-1}, 2 k, 0 ; \tau\right)=\frac{1}{(n-1) !}\left(\tau I_{n-1}\left(G_{2 k} ; \tau\right)-I_{n}\left(G_{2 k} ; \tau\right)\right) .
$$

Proof The first equality is immediate from the definition (2.2). The second equality (2.5) is trivial for $n=0$, and the general case is easy to prove from (2.4) by induction on $n$. Finally, (2.6) follows directly from (2.4), (2.5) and the definition (2.3). 


\subsection{The elliptic KZB connection and the associated transport map}

We recall the definition of the elliptic KZB (Knizhnik-Zamolodchikov-Bernard) connection $\nabla_{\mathrm{KZB}}$ on $E_{\tau}^{\times}$, whose monodromy will give rise to the elliptic KZB associator. Originally, $\nabla_{\text {KZB }}$ was defined as a meromorphic connection on $\mathbb{C}$ (cf. [9, 17, 23]). Here, we will instead follow [8], which consider a certain $C^{\infty}$-trivialization of $\nabla_{\mathrm{KZB}}$, which is defined on the quotient $\mathbb{C} /(\mathbb{Z}+\mathbb{Z} \tau) \backslash\{0\}$.

Let $\xi=r \tau+s$ be the canonical coordinate on $E_{\tau}^{\times}$, with $(r, s) \in \mathbb{R}^{2} \backslash \mathbb{Z}^{2}$. Also, let

$$
\theta_{\tau}(\xi)=\sum_{n \in \mathbb{Z}}(-1)^{n} q^{\frac{1}{2}\left(n+\frac{1}{2}\right)^{2}} e^{\left(n+\frac{1}{2}\right) \xi}, \quad q=e^{2 \pi i \tau},
$$

be the classical Jacobi theta function.

Definition 2.4 (Brown-Levin, Calaque-Enriquez-Etingof, Levin-Racinet) Define a connection $\nabla_{\mathrm{KZB}}$ on the trivial bundle ${ }^{2}$

$$
E_{\tau}^{\times} \times \mathbb{C}\langle\langle\mathrm{a}, \mathrm{b}\rangle\rangle \rightarrow E_{\tau}^{\times}
$$

by setting $\nabla_{\mathrm{KZB}}(f):=\mathrm{d} f-\omega_{\mathrm{KZB}} \cdot f$ for a local section $f$, where

$$
\omega_{\mathrm{KZB}}=\mathrm{d} r \cdot \mathrm{a}+2 \pi i \operatorname{ad}(\mathrm{a}) e^{r \operatorname{ad}(\mathrm{a})} F_{\tau}(2 \pi i \xi, \operatorname{ad}(\mathrm{a}))(\mathrm{b}) \mathrm{d} \xi,
$$

where

$$
F_{\tau}(\xi, \eta):=\frac{\theta_{\tau}^{\prime}(0) \theta_{\tau}(\xi+\eta)}{\theta_{\tau}(\xi) \theta_{\tau}(\eta)} .
$$

Proposition 2.5 The connection $\nabla_{\mathrm{KZB}}$ satisfies the following properties.

(i) We have $\nabla_{\mathrm{KZB}}^{2}=0$; in other words, $\nabla_{\mathrm{KZB}}$ is integrable.

(ii) The connection $\nabla_{\mathrm{KZB}}$ has a simple pole at $\xi=0$ with residue

$$
\operatorname{Res}_{0}\left(\nabla_{\mathrm{KZB}}\right)=[\mathrm{a}, \mathrm{b}]
$$

Proof (i) The condition $\nabla_{\mathrm{KZB}}^{2}=0$ is equivalent to

$$
\mathrm{d} \omega_{\mathrm{KZB}}-\omega_{\mathrm{KZB}} \wedge \omega_{\mathrm{KZB}}=0,
$$

which in turn follows from a direct computation:

$$
\begin{aligned}
\mathrm{d} \omega_{\mathrm{KZB}} & =2 \pi i \mathrm{~d} r \cdot \operatorname{ad}(\mathrm{a}) \wedge \operatorname{ad}(\mathrm{a}) e^{r \operatorname{ad}(\mathrm{a})} F_{\tau}(2 \pi i \xi, \operatorname{ad}(\mathrm{a}))(\mathrm{b}) \mathrm{d} \xi \\
& =\omega_{\mathrm{KZB}} \wedge \omega_{\mathrm{KZB}} .
\end{aligned}
$$

\footnotetext{
2 Note that the normalization of the variables $\mathrm{a}, \mathrm{b}$ differs from [8], Example 5.3.1, by $\mathrm{a}=-2 \pi i \mathrm{x}_{0}$ and $\mathrm{b}=-(2 \pi i)^{-1} \mathrm{x}_{1}$. Our conventions are compatible with [17], §11.1.
} 
(ii) The residue of the connection $\nabla_{\mathrm{KZB}}$ is just the residue of the one-form $\omega_{\mathrm{KZB}}$. But the computation of the latter is easy from the definition, using the fact that the residue of $2 \pi i F_{\tau}(2 \pi i \xi, \eta)$ at $\xi=0$ is equal to one (cf. [17], eqn.(8)).

Now for any two base points $\rho_{1}, \rho_{2}$, let $\pi_{1}\left(E_{\tau}^{\times} ; \rho_{2}, \rho_{1}\right)$ be the fundamental torsor of paths from $\rho_{1}$ to $\rho_{2}$. The integrability of $\nabla_{\mathrm{KZB}}$ implies that the transport function

$$
\begin{aligned}
T_{\rho_{2}, \rho_{1}}^{\mathrm{KZB}}: \pi_{1}\left(E_{\tau}^{\times} ; \rho_{2}, \rho_{1}\right) & \rightarrow \mathbb{C}\langle\langle\mathrm{a}, \mathrm{b}\rangle\rangle \\
\gamma & \mapsto \sum_{k=0}^{\infty} \int_{\gamma} \omega_{\mathrm{KZB}}^{k},
\end{aligned}
$$

is well-defined, where $\int_{\gamma} \omega_{\mathrm{KZB}}^{k}$ denotes the iterated integral in the sense of Chen [10]

$$
\int_{\gamma} \omega_{\mathrm{KZB}}^{k}:=\int_{1 \geq t_{1} \geq \ldots \geq t_{k} \geq 1} \gamma^{*}\left(\omega_{\mathrm{KZB}}\right)\left(t_{1}\right) \ldots \gamma^{*}\left(\omega_{\mathrm{KZB}}\right)\left(t_{k}\right) .
$$

In other words, $\int_{\gamma} \omega_{\mathrm{KZB}}^{k}$ depends only on the homotopy class of $\gamma$.

Rather than choosing points $\rho_{1}, \rho_{2} \in E_{\tau}^{\times}$, which is not canonical, we work with tangential base points, in the sense of [11], $\$ 15$, at the puncture 0 . Since $\nabla_{\mathrm{KZB}}$ has only a simple pole at $\xi=0$, one can extend the definition of the transport function to the case of tangential base points as in [11], Proposition 15.45. More precisely, for any two non-zero tangent vectors $\vec{v}_{0}=\lambda \frac{\partial}{\partial \xi}$ and $\vec{w}_{0}=\mu \frac{\partial}{\partial \xi}$ at 0 , there is a well-defined function

$$
T_{\vec{w}_{0}, \vec{v}_{0}}^{\mathrm{KZB}}: \pi_{1}\left(E_{\tau}^{\times} ; \vec{w}_{0}, \vec{v}_{0}\right) \rightarrow \mathbb{C}\langle\langle\mathrm{a}, \mathrm{b}\rangle\rangle
$$

given by

$$
T_{\vec{w}_{0}, \vec{v}_{0}}^{\mathrm{KZB}}(\gamma)=\lim _{t \rightarrow 0} e^{\log \left(\mu^{-1} t\right) \operatorname{Res}_{0}\left(\nabla_{\mathrm{KZB}}\right)}\left[\sum_{k=0}^{\infty} \int_{\gamma_{t}^{1-t}} \omega_{\mathrm{KZB}}^{k}\right] e^{-\log \left(\lambda^{-1} t\right) \operatorname{Res}_{0}\left(\nabla_{\mathrm{KZB}}\right)},
$$

where $\operatorname{Res}_{0}\left(\nabla_{\mathrm{KZB}}\right)=[\mathrm{a}, \mathrm{b}]$ is the residue of the connection at $\xi=0$ (cf. Proposition 2.5.(i)), $\gamma_{t}^{1-t}$ denotes the restriction of $\gamma$ to the interval $[t, 1-t]$ (for $0<t<\frac{1}{2}$ ) and the branches of the logarithms are determined by the path $\gamma$. For arithmetic applications, it will be important that the tangent vectors are integral on the Tate curve $\mathbb{C}^{\times} / q^{\mathbb{Z}}$ and moreover non-zero modulo every prime number $p$, which fixes them uniquely (up to a sign): $\vec{v}_{0}= \pm \frac{\partial}{\partial z}= \pm(2 \pi i)^{-1} \frac{\partial}{\partial \xi}$, where $z=e^{2 \pi i \xi}$.

\section{The elliptic depth filtration}

We recall the definition of the elliptic depth filtration on the fundamental Lie algebra of $E_{\tau}^{\times}$(cf. [19], §27). This filtration is the elliptic analog of the depth filtration on the fundamental Lie algebra of $\mathbb{P}^{1} \backslash\{0,1, \infty\}([12], \S 6$ or [5], §4). 


\subsection{The elliptic depth filtration}

Consider the canonical embedding

$$
E_{\tau}^{\times} \hookrightarrow E_{\tau}
$$

of the once-punctured elliptic curve $E_{\tau}^{\times}$into the (complete) elliptic curve $E_{\tau}$. On fundamental Lie algebras, it induces the abelianization map

$$
\pi: \mathfrak{p}\left(E_{\tau}^{\times}\right) \rightarrow \mathfrak{p}\left(E_{\tau}^{\times}\right)^{\mathrm{ab}} \cong \mathfrak{p}\left(E_{\tau}\right) .
$$

Definition 3.1 (Hain-Matsumoto) The elliptic depth filtration $D^{\bullet} \mathfrak{p}\left(E_{\tau}^{\times}\right)$is the descending filtration on $\mathfrak{p}\left(E_{\tau}^{\times}\right)$, defined by

$$
D^{n} \mathfrak{p}\left(E_{\tau}^{\times}\right)=\left\{\begin{array}{ll}
\mathfrak{p}\left(E_{\tau}^{\times}\right) & n=0 \\
\operatorname{ker}(\pi) & n=1 . \\
{\left[D^{1} \mathfrak{p}\left(E_{\tau}^{\times}\right), D^{n-1} \mathfrak{p}\left(E_{\tau}^{\times}\right)\right]} & n \geq 2
\end{array} .\right.
$$

Also, let $\operatorname{gr}_{D}^{\bullet} \mathfrak{p}\left(E_{\tau}^{\times}\right)$be the associated graded Lie algebra.

It is clear from the definition that the elliptic depth filtration is the lower central series on the commutator of $\mathfrak{p}\left(E_{\tau}^{\times}\right)$. Therefore, the quotient Lie algebra

$$
\mathfrak{p}\left(E_{\tau}^{\times}\right)^{\text {met-ab }}:=\mathfrak{p}\left(E_{\tau}^{\times}\right) / D^{2} \mathfrak{p}\left(E_{\tau}^{\times}\right)
$$

is the (maximal) meta-abelian quotient of $\mathfrak{p}\left(E_{\tau}^{\times}\right)$.

The following proposition is well-known.

Proposition 3.2 We have isomorphisms of (abelian) Lie algebras

$$
\operatorname{gr}_{D}^{0} \mathfrak{p}\left(E_{\tau}^{\times}\right) \cong \mathbb{Q} \mathrm{a} \oplus \mathbb{Q} \mathrm{b}
$$

and

$$
\begin{aligned}
\operatorname{gr}_{D}^{1} \mathfrak{p}\left(E_{\tau}^{\times}\right) & \stackrel{\cong}{\mathbb{Q} \llbracket U, \mathrm{~V} \rrbracket} \\
\operatorname{ad}^{k}(\mathrm{a}) \operatorname{ad}^{l}(\mathrm{~b})([\mathrm{a}, \mathrm{b}]) & \mapsto \mathrm{U}^{k} \mathrm{~V}^{l} .
\end{aligned}
$$

Moreover,

$$
\mathfrak{p}\left(E_{\tau}^{\times}\right)^{\mathrm{met}-\mathrm{ab}} \cong \operatorname{gr}_{D}^{0} \mathfrak{p}\left(E_{\tau}^{\times}\right) \ltimes \operatorname{gr}_{D}^{1} \mathfrak{p}\left(E_{\tau}^{\times}\right)
$$

as Lie algebras, where $\mathbb{Q} \mathbf{a} \oplus \mathbb{Q} \mathrm{b}$ acts on $\operatorname{gr}_{D}^{1} \mathfrak{p}\left(E_{\tau}^{\times}\right) \cong \mathbb{Q} \llbracket \mathrm{U}, \mathrm{V} \rrbracket$ by the adjoint action.

Proof The first isomorphism is clear, since the right hand side of (3.1) is just the abelianization of $\mathfrak{p}\left(E_{\tau}^{\times}\right)$. It follows from the Jacobi identity that every element of $\operatorname{gr}_{D}^{1} \mathfrak{p}\left(E_{\tau}^{\times}\right)$is a series in the elements $\operatorname{ad}^{k}(\mathrm{a}) \operatorname{ad}^{l}(\mathrm{~b})([\mathrm{a}, \mathrm{b}])$, and then the isomorphism 
(3.2) is a consequence of the universal property of free Lie algebras. Finally, the last statement of the proposition follows from the fact that the adjoint action splits the short exact sequence of Lie algebras

$$
0 \longrightarrow \operatorname{gr}_{D}^{1} \mathfrak{p}\left(E_{\tau}^{\times}\right) \longrightarrow \mathfrak{p}\left(E_{\tau}^{\times}\right) / D^{2} \mathfrak{p}\left(E_{\tau}^{\times}\right) \longrightarrow \operatorname{gr}_{D}^{0} \mathfrak{p}\left(E_{\tau}^{\times}\right) \longrightarrow 0 .
$$

Remark 3.3 The relation between the elliptic depth filtration and the depth filtration on the fundamental Lie algebra of $\mathbb{P}^{1} \backslash\{0,1, \infty\}$ can be explained as follows. First, recall (cf. [12], §5) that the (de Rham) fundamental Lie algebra $\mathfrak{p}(U)$ of $U:=\mathbb{P}^{1} \backslash\{0,1, \infty\}$ is isomorphic to $\mathbb{L}\left(\mathbf{x}_{0}, \mathbf{x}_{1}\right)^{\wedge}$. The depth filtration $D^{n} \mathfrak{p}(U)$ on $\mathfrak{p}(U)$ is then the lower central series on the kernel of the natural map between fundamental Lie algebras

$$
\begin{aligned}
\mathfrak{p}(U) & \rightarrow \mathbb{L}\left(\mathrm{x}_{0}\right)^{\wedge} \cong \mathbb{Q} \mathrm{x}_{0} \\
\mathrm{x}_{i} & \mapsto \delta_{i, 0} \mathrm{x}_{0},
\end{aligned}
$$

which is induced from the embedding $\mathbb{P}^{1} \backslash\{0,1, \infty\} \hookrightarrow \mathbb{P}^{1} \backslash\{0, \infty\}$ (cf. [5, 12]). Interpreting $\mathbb{P}^{1} \backslash\{0,1, \infty\}$ as the fiber over $q=0$ of the universal once-punctured Tate curve $\left(\mathbb{C}^{\times} / q^{\mathbb{Z}}\right) \backslash\{1\}$, one obtains a morphism of Lie algebras $[7,14,17]$

$$
\begin{aligned}
\iota: \mathfrak{p}(U) & \rightarrow \mathfrak{p}\left(E_{\tau}^{\times}\right) \\
\mathrm{x}_{0} & \mapsto \frac{\operatorname{ad}(\mathrm{a})}{e^{\operatorname{ad}(\mathrm{a})}-1}(\mathrm{~b})=\sum_{k=0}^{\infty} \frac{B_{k}}{k !} \mathrm{ad}^{k}(\mathrm{a})(\mathrm{b}) \\
\mathrm{x}_{1} & \mapsto[\mathrm{a}, \mathrm{b}],
\end{aligned}
$$

which clearly respects the depth filtrations on both sides, i.e.

$$
\iota\left(D^{n} \mathfrak{p}(U)\right)=\iota(\mathfrak{p}(U)) \cap D^{n} \mathfrak{p}\left(E_{\tau}^{\times}\right), \quad \text { for all } n \geq 0 .
$$

For more details, see [19], §27.

\subsection{Action of special derivations in depths zero and one}

We now compute the action of the derivations $\varepsilon_{2 k}$ on the meta-abelian quotient $\mathfrak{p}\left(E_{\tau}^{\times}\right)^{\text {met-ab }}$.

Proposition 3.4 (i) The derivation $\varepsilon_{0}$ acts on $\operatorname{gr}_{D}^{0} \mathfrak{p}\left(E_{\tau}^{\times}\right) \cong \mathbb{Q} \mathrm{a} \oplus \mathbb{Q b}$ as the linear map $\left(\begin{array}{cc}0 & -1 \\ 0 & 0\end{array}\right)$, and on $\operatorname{gr}_{D}^{1} \mathfrak{p}\left(E_{\tau}^{\times}\right) \cong \mathbb{Q} \llbracket \mathrm{U}, \mathrm{V} \rrbracket$ as the derivation $-\mathrm{V} \frac{\partial}{\partial \mathrm{U}}$.

(ii) The derivations $\varepsilon_{2 k}$, for $k>0$, act trivially on $\operatorname{gr}_{D}^{i} \mathfrak{p}\left(E_{\tau}^{\times}\right)$, for every $i \geq 0$.

(iii) Let $\underline{2 k}=\left(2 k_{1}, \ldots, 2 k_{n}\right)$ be a multi-index, where $k_{i} \geq 0$. Then $\varepsilon_{2 k}=\varepsilon_{2 k_{1}} \circ \cdots \circ$ $\varepsilon_{2 k_{n}}$ acts non-trivially on $\mathfrak{p}\left(E_{\tau}^{\times}\right)^{\text {met }-\mathrm{ab}} \cong \operatorname{gr}_{D}^{0} \mathfrak{p}\left(E_{\tau}^{\times}\right) \ltimes \mathrm{gr}_{D}^{1} \mathfrak{p}\left(E_{\tau}^{\overline{\times}}\right)$, only if either $\underline{2 k}=\left(0, \ldots, 0,2 k_{n}\right)$ or $\underline{2 k}=\left(0, \ldots, 0,2 k_{n-1}, 0\right)$. 
Proof The action of $\varepsilon_{0}$ on $\operatorname{gr}_{D}^{0} \mathfrak{p}\left(E_{\tau}^{\times}\right)$is clear from the definition (cf. Definition 2.1). For the action on $\operatorname{gr}_{D}^{1} \mathfrak{p}\left(E_{\tau}^{\times}\right)$, by the Jacobi identity, the linear operators ad(a), ad(b) $\in$ $\operatorname{End}\left(\operatorname{gr}_{D}^{1} \mathfrak{p}\left(E_{\tau}^{\times}\right)\right)$commute with each other. Consequently, we have

$$
\begin{aligned}
\varepsilon_{0}\left(\operatorname{ad}^{k}(\mathrm{a}) \operatorname{ad}^{l}(\mathrm{~b})([\mathrm{a}, \mathrm{b}])\right) & \equiv \sum_{i=0}^{k-1}-\operatorname{ad}^{i}(\mathrm{a}) \operatorname{ad}(\mathrm{b}) \operatorname{ad}^{k-1-i}(\mathrm{a}) \operatorname{ad}^{l}(\mathrm{~b})([\mathrm{a}, \mathrm{b}]) \\
& \equiv-k \operatorname{ad}^{k-1}(\mathrm{a}) \operatorname{ad}^{l+1}(\mathrm{~b})([\mathrm{a}, \mathrm{b}]) \quad \bmod D^{2} \mathfrak{p}\left(E_{\tau}^{\times}\right)
\end{aligned}
$$

Therefore, under the isomorphism $\operatorname{gr}_{D}^{1} \mathfrak{p}\left(E_{\tau}^{\times}\right) \cong \mathbb{Q} \llbracket \mathrm{U}, \mathrm{V} \rrbracket$ of Proposition 3.2, the derivation $\varepsilon_{0}$ corresponds to $-\mathrm{V} \frac{\partial}{\partial U}$. As for (ii), the triviality of $\varepsilon_{2 k}$, for $k>0$, on $\operatorname{gr}_{D}^{0} \mathfrak{p}\left(E_{\tau}^{\times}\right)$is clear from Definition 2.1, and triviality on $\operatorname{gr}_{D}^{i} \mathfrak{p}\left(E_{\tau}^{\times}\right)$follows by induction on $i$. Finally, (iii) follows easily from (i) and (ii).

\section{The elliptic KZB associator}

In this section, we define Enriquez's elliptic KZB associator [14], which is an elliptic analogue of the Drinfeld associator [13]. Our approach differs slightly from [14] in that we define the elliptic KZB associator using the "elliptic transport isomorphism" of Brown-Levin. This definition is analogous to the definition of the Drinfeld associator using parallel transport along the KZ-connection [12]. We also recall an important result of Enriquez (cf. [14], §6) which describes the variation of the elliptic KZB associator in the modulus of the once-punctured elliptic curve.

\subsection{Definition via the transport function}

In Sect. 2.4, we have defined a transport function $T_{\rho_{2}, \rho_{1}}^{\mathrm{KZB}}$ on a once-punctured elliptic curve for any choice of base points $\rho_{1}, \rho_{2}$ (possibly tangential), using the elliptic KZB connection. We now specialize these base points to be $\pm \vec{v}_{0}$, where $\vec{v}_{0}$ is the tangent vector $-(2 \pi i)^{-1} \frac{\partial}{\partial \xi}$ at $0 \in E_{\tau}$. Note that under the isomorphism $E_{\tau} \cong \mathbb{C}^{\times} / q^{\mathbb{Z}}$, we have $\vec{v}_{0}=-\frac{\partial}{\partial z}$, where $z=e^{2 \pi i \xi}$. In particular, $\vec{v}_{0}$ is defined over $\mathbb{Z}$ on the Tate curve.

Consider now the paths $\alpha, \beta \in \pi_{1}\left(E_{\tau}^{\times} ;-\vec{v}_{0}, \vec{v}_{0}\right)$ which are the images of, respectively, the (open) straight-line paths $(0,1)$ and $(0, \tau)$ under the projection $\mathbb{C} \backslash(\mathbb{Z}+\mathbb{Z} \tau) \rightarrow E_{\tau}^{\times}$, where the path $(0, \tau)$ is additionally composed with a half-circle in the positive direction around $\tau$. Therefore (after ignoring the $-(2 \pi i)^{-1}$-prefactor), the paths $\alpha, \beta$ look like in Fig. 1 below (cf. [14], p.550).

Definition 4.1 ([14], §6.2) The elliptic KZB associator is the tuple $(A(\tau), B(\tau))$, where

$$
A(\tau):=T_{-\vec{v}_{0}, \vec{v}_{0}}^{\mathrm{KZB}}(\alpha), \quad B(\tau):=T_{-\vec{v}_{0}, \vec{v}_{0}}^{\mathrm{KZB}}(\beta)
$$

are the images of the paths $\alpha$ and $\beta$ under the transport map $T_{-\vec{v}_{0}, \vec{v}_{0}}^{\mathrm{KZB}}$. 
Fig. 1 The paths $\alpha$ and $\beta$

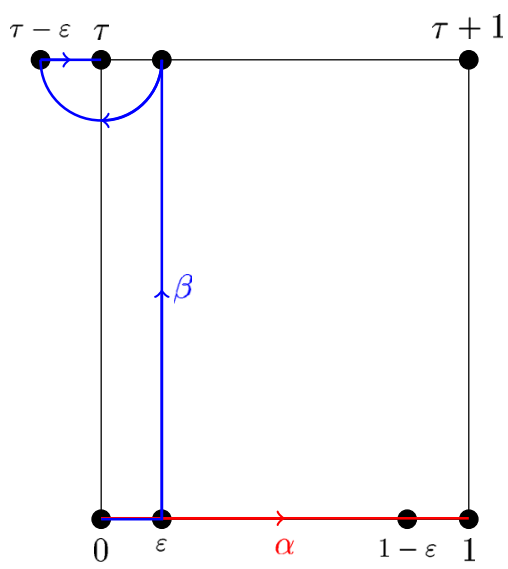

Remark 4.2 The definition of the elliptic KZB associator given here is not exactly the same as the one given in [14], but equivalent. Using the elliptic transport map, Enriquez definition is

$$
A^{\mathrm{Enr}}(\tau):=T_{\vec{v}_{0}}^{\mathrm{KZB}}(\alpha), \quad B^{\mathrm{Enr}}(\tau):=T_{\vec{v}_{0}}^{\mathrm{KZB}}(\beta) .
$$

Explicitly, the relation between the two versions is given by

$$
A(\tau)=e^{-\pi i[\mathrm{a}, \mathrm{b}]} A^{\mathrm{Enr}}(\tau), \quad B(\tau)=e^{\pi i[\mathrm{a}, \mathrm{b}]} B^{\mathrm{Enr}}(\tau) .
$$

\subsection{Variation in the modulus}

An important property of the elliptic KZB associator is that it satisfies a linear differential equation, which relates it to iterated Eisenstein integrals and the special derivations $\varepsilon_{2 k}$ reviewed in Sect. 2. The boundary condition of this differential equation establishes a relation between the series $A(\tau), B(\tau)$ and the Drinfeld associator $\Phi$. More precisely, we have the following theorem, due to Enriquez.

Theorem 4.3 ([15], §5.2) We have

$$
A(\tau)=g(\tau)\left(A_{\infty}\right), \quad B(\tau)=g(\tau)\left(B_{\infty}\right),
$$

where

$$
g(\tau)=\sum(-2 \pi i)^{n} \mathcal{G}\left(2 k_{1}, \ldots, 2 k_{n} ; \tau\right) \cdot\left(\varepsilon_{2 k_{1}} \circ \ldots \circ \varepsilon_{2 k_{n}}\right),
$$

the sum being over all multi-indices $\left(k_{1}, \ldots, k_{n}\right) \in \mathbb{Z}_{\geq 0}^{n}$, for $n \geq 0$, and

$$
\begin{aligned}
& \left.A_{\infty}=e^{\pi i \iota\left(\mathbf{x}_{1}\right)} \Phi\left(\iota\left(\mathbf{x}_{0}\right)\right), \iota\left(\mathbf{x}_{1}\right)\right) e^{2 \pi i \iota\left(\mathbf{x}_{0}\right)} \Phi\left(\iota\left(\mathbf{x}_{0}\right), \iota\left(\mathbf{x}_{1}\right)\right)^{-1}, \\
& \left.B_{\infty}=\Phi\left(\iota\left(\mathbf{x}_{\infty}\right)\right), \iota\left(\mathbf{x}_{1}\right)\right) e^{a} \Phi\left(\iota\left(\mathbf{x}_{0}\right), \iota\left(\mathbf{x}_{1}\right)\right)^{-1},
\end{aligned}
$$

where $\iota: \mathfrak{p}(U) \rightarrow \mathfrak{p}\left(E_{\tau}^{\times}\right)$is the morphism of Remark 3.3. 
The element $g(\tau)$ defines an automorphism of $\exp \mathfrak{p}\left(E_{\tau}^{\times}\right)$. Letting

$$
\begin{aligned}
& \mathfrak{A}(\tau):=\log (A(\tau)), \quad \mathfrak{B}(\tau):=\log (B(\tau)), \\
& \mathfrak{A}_{\infty}:=\log \left(A_{\infty}\right), \quad \mathfrak{B}_{\infty} \quad:=\log \left(B_{\infty}\right),
\end{aligned}
$$

we also have

$$
\mathfrak{A}(\tau)=g(\tau)\left(\mathfrak{A}_{\infty}\right), \quad \mathfrak{B}(\tau)=g(\tau)\left(\mathfrak{B}_{\infty}\right),
$$

since $g(\tau)$ commutes with exponential and logarithm functions.

The next corollary follows immediately from Proposition 3.4.

Corollary 4.4 Let $g(\tau)^{\text {met-ab }}$ be the image of $g(\tau)$ in $\operatorname{End}\left(\mathfrak{p}\left(E_{\tau}^{\times}\right)_{\mathbb{C}}^{\text {met-ab }}\right)$. We have

$$
\begin{aligned}
g(\tau)^{\mathrm{met}-\mathrm{ab}}= & \sum_{n \geq 0}(-2 \pi i)^{n} \mathcal{G}\left(\{0\}_{n} ; \tau\right) \cdot \varepsilon_{0}^{n} \\
& +\sum_{n \geq 0, k \geq 1}(-2 \pi i)^{n+1} \mathcal{G}\left(\{0\}_{n}, 2 k ; \tau\right) \cdot\left(\varepsilon_{0}^{n} \circ \varepsilon_{2 k}\right) \\
& +\sum_{k, n \geq 1}(-2 \pi i)^{n+1} \mathcal{G}\left(\{0\}_{n-1}, 2 k, 0 ; \tau\right) \cdot\left(\varepsilon_{0}^{n-1} \circ \varepsilon_{2 k} \circ \varepsilon_{0}\right) .
\end{aligned}
$$

Remark 4.5 The pair $\left(A_{\infty}, B_{\infty}\right)$ is the image of the Drinfeld associator under the natural map ([14], §4.5)

$$
\underline{M}(\mathbb{C}) \rightarrow \underline{E l l}(\mathbb{C}),
$$

where $\underline{M}$ is the scheme of classical associators in the sense of [13], and Ell is its elliptic counterpart [14]. A geometric way of interpreting this morphism is via the degeneration of the once-punctured Tate curve to $\mathbb{P}^{1} \backslash\{0,1, \infty\}$ (cf. Remark 3.3).

\subsection{Elliptic KZB associator in depth zero}

Let $\mathfrak{A}(\tau)^{0}$ be the image of $\mathfrak{A}(\tau)$ in $\operatorname{gr}_{D}^{0} \mathfrak{p}\left(E_{\tau}^{\times}\right) \mathbb{C}=\mathfrak{p}\left(E_{\tau}^{\times}\right) /\left[\mathfrak{p}\left(E_{\tau}^{\times}\right), \mathfrak{p}\left(E_{\tau}^{\times}\right)\right]$, and likewise let $\mathfrak{B}(\tau)^{(0)}$ be the image of $\mathfrak{B}(\tau)$ in $\operatorname{gr}_{D}^{0} \mathfrak{p}\left(E_{\tau}^{\times}\right) \mathbb{C}$.

The following proposition shows that $\mathfrak{A}(\tau)^{0}$ and $\mathfrak{B}(\tau)^{0}$ precisely retrieve the periods of $H^{1}\left(E_{\tau}^{\times}\right)$.

\section{Proposition 4.6 We have}

$$
\mathfrak{A}(\tau)^{(0)}=2 \pi i \mathrm{~b}, \quad \mathfrak{B}(\tau)^{(0)}=\mathrm{a}+2 \pi i \tau \mathrm{b} .
$$

Proof We only prove the result for $\mathfrak{A}(\tau)^{(0)}$, the formula for $\mathfrak{B}(\tau)^{(0)}$ is proved analogously. By Theorem 4.3, we know that $A(\tau)=g(\tau)\left(A_{\infty}\right)$, and since $g(\tau)$ is an automorphism, we also have

$$
\mathfrak{A}(\tau)=\log (A(\tau))=g(\tau)\left(\log \left(A_{\infty}\right)\right)=g(\tau)\left(\mathfrak{A}_{\infty}\right) .
$$


On the other hand, it follows directly from the explicit formula for $A_{\infty}$ given in Theorem 4.3 that

$$
\mathfrak{A}_{\infty} \equiv 2 \pi i \mathrm{~b} \bmod D^{1} \mathfrak{p}\left(E_{\tau}^{\times}\right),
$$

since $\iota\left(\mathbf{x}_{0}\right) \equiv \mathrm{b} \bmod D^{1} \mathfrak{p}\left(E_{\tau}^{\times}\right)$and $\iota\left(\mathbf{x}_{1}\right) \equiv 0 \bmod D^{1} \mathfrak{p}\left(E_{\tau}^{\times}\right)$. But as every derivation $\varepsilon_{2 k}$ annihilates $\mathrm{b}$, we finally get $\mathfrak{A}(\tau)^{(0)}=g(\tau)(2 \pi i \mathrm{~b})=2 \pi i \mathrm{~b}$.

Remark 4.7 Proposition 4.6 could have also been proved directly without recourse to Enriquez' Theorem 4.3, using that $\omega_{\mathrm{KZB}} \equiv \mathrm{d} r \cdot \mathrm{a}+2 \pi i \mathrm{~d} \xi \cdot \mathrm{b} \bmod D^{1} \mathfrak{p}\left(E_{\tau}^{\times}\right)$.

\section{The meta-abelian elliptic KZB associator}

In this section, we compute the image of $\mathfrak{A}(\tau)$ and $\mathfrak{B}(\tau)$ in the meta-abelian quotient $\mathfrak{p}\left(E_{\tau}^{\times}\right)_{\mathbb{C}}^{\text {met-ab }}$ of $\mathfrak{p}\left(E_{\tau}^{\times}\right) \mathbb{C}$. The strategy is to use Theorem 4.3 which yields that

$$
\mathfrak{A}(\tau)=g(\tau)\left(\mathfrak{A}_{\infty}\right), \quad \mathfrak{B}(\tau)=g(\tau)\left(\mathfrak{B}_{\infty}\right)
$$

and then to compute the images of $\mathfrak{A}_{\infty}$ and $\mathfrak{B}_{\infty}$ in the meta-abelian quotient separately. This is done in Sect. 5.1. In Sect. 5.2, we then compute the action of $g(\tau)$ on the metaabelian quotient. The two computations are then combined in Sect. 5.3 to yield our formula for $\mathfrak{A}(\tau)^{\text {met-ab }}$ and $\mathfrak{B}(\tau)^{\text {met-ab }}$.

\subsection{The arithmetic piece: periods of Eisenstein series}

Let $\mathfrak{A}_{\infty}^{\text {met-ab }}$ (resp. $\mathfrak{B}_{\infty}^{\text {met-ab }}$ ) be the image of $\mathfrak{A}_{\infty}$ (resp. the image of $\mathfrak{B}_{\infty}$ ) in the meta-abelian quotient $\mathfrak{p}\left(E_{\tau}^{\times}\right)_{\mathbb{C}}^{\text {met-ab }} \cong \operatorname{gr}_{D}^{0} \mathfrak{p}\left(E_{\tau}^{\times}\right) \mathbb{C} \ltimes \operatorname{gr}_{D}^{1} \mathfrak{p}\left(E_{\tau}^{\times}\right)_{\mathbb{C}}$, so that we can write

$$
\mathfrak{A}_{\infty}^{\text {met-ab }}=\mathfrak{A}_{\infty}^{(0)}+\mathfrak{A}_{\infty}^{(1)}, \quad \mathfrak{B}_{\infty}^{\text {met-ab }}=\mathfrak{B}_{\infty}^{(0)}+\mathfrak{B}_{\infty}^{(1)} .
$$

The computation of the depth zero component was already carried out in Proposition 4.6 so that it remains to compute the depth one contribution. For this, we need a short lemma about the Drinfeld associator.

Lemma 5.1 Let $\varphi\left(\mathbf{x}_{0}, \mathbf{x}_{1}\right):=\log \left(\Phi\left(\mathbf{x}_{0}, \mathbf{x}_{1}\right)\right)$. Then

$$
\varphi\left(\iota\left(\mathbf{x}_{0}\right), \iota\left(\mathbf{x}_{1}\right)\right) \equiv-\sum_{n \geq 2} \zeta(n) \operatorname{ad}^{n-1}(\mathrm{~b})([\mathrm{a}, \mathrm{b}]) \quad \bmod D^{2} \mathfrak{p}\left(E_{\tau}^{\times}\right) \mathbb{C}
$$

where $\iota\left(\mathrm{x}_{0}\right)=\frac{\mathrm{ad}(\mathrm{a})}{e^{\mathrm{ad}(\mathrm{a})}-1}(\mathrm{~b})$ and $\iota\left(\mathrm{x}_{1}\right)=[\mathrm{a}, \mathrm{b}]($ cf. Remark 3.3). In particular, we have $\varphi\left(\iota\left(\mathbf{x}_{0}\right), \iota\left(\mathbf{x}_{1}\right)\right) \in D^{1} \mathfrak{p}\left(E_{\tau}^{\times}\right) \mathbb{C}$.

Proof It is well-known (cf. [12], §6.7) that

$$
\varphi\left(\mathbf{x}_{0}, \mathbf{x}_{1}\right) \equiv-\sum_{n=2}^{\infty} \zeta(n) \operatorname{ad}^{n-1}\left(\mathbf{x}_{0}\right)\left(\mathbf{x}_{1}\right)
$$

Applying $\iota$ to both sides, we get the result. 
Theorem 5.2 We have

$$
\begin{aligned}
\mathfrak{A}_{\infty}^{(1)} & =2 \pi i\left(c(\mathrm{U})-\frac{2 \pi i}{4} \mathrm{~V}+\sum_{n \geq 3, \text { odd }} \zeta(n) \mathrm{V}^{n}\right), \\
\mathfrak{B}_{\infty}^{(1)} & =-2 \pi i(c(2 \pi i \mathrm{~V})-\mathrm{U} c(\mathrm{U}) c(2 \pi i \mathrm{~V}))+\sum_{n \geq 3, \text { odd }} \zeta(n) \mathrm{UV}^{n-1},
\end{aligned}
$$

where $c(x)=\frac{1}{e^{x}-1}+\frac{1}{2}-\frac{1}{x}=\sum_{k=2}^{\infty} \frac{B_{k}}{k !} x^{k-1}$.

Proof By Theorem 4.3, we know that

$$
\mathfrak{A}_{\infty}=\log \left(e^{\pi i \iota\left(\mathbf{x}_{1}\right)} \Phi\left(\iota\left(\mathbf{x}_{0}\right), \iota\left(\mathbf{x}_{1}\right)\right) e^{2 \pi i \iota\left(\mathbf{x}_{0}\right)} \Phi\left(\iota\left(\mathbf{x}_{0}\right), \iota\left(\mathbf{x}_{1}\right)\right)^{-1}\right) .
$$

Using a "truncated" version of the Baker-Campbell-Hausdorff formula (cf. [30], Corollary 3.24) and Lemma 5.1, we get

$$
\begin{aligned}
\mathfrak{S} & :=\log \left(e^{\pi i \iota\left(\mathbf{x}_{1}\right)} \Phi\left(\iota\left(\mathbf{x}_{0}\right), \iota\left(\mathbf{x}_{1}\right)\right)\right) \\
& \equiv \varphi\left(\iota\left(\mathbf{x}_{0}\right), \iota\left(\mathbf{x}_{1}\right)\right)+\sum_{k \geq 0} \frac{B_{k}}{k !} \operatorname{ad}^{k}\left(\varphi\left(\iota\left(\mathbf{x}_{0}\right), \iota\left(\mathbf{x}_{1}\right)\right)\right)\left(\pi i \iota\left(\mathbf{x}_{1}\right)\right) \\
& \equiv \varphi\left(\iota\left(\mathbf{x}_{0}\right), \iota\left(\mathbf{x}_{1}\right)\right)+\pi i \iota\left(\mathbf{x}_{1}\right) \quad \bmod D^{2} \mathfrak{p}\left(E_{\tau}^{\times}\right) \mathbb{C} .
\end{aligned}
$$

Similarly, since $\iota\left(\mathbf{x}_{0}\right) \equiv b \bmod D^{1} \mathfrak{p}\left(E_{\tau}^{\times}\right)_{\mathbb{C}}$, we get

$$
\begin{aligned}
\mathfrak{T} & :=\log \left(e^{2 \pi i \iota\left(\mathbf{x}_{0}\right)} \Phi\left(\iota\left(\mathbf{x}_{0}\right), \iota\left(\mathbf{x}_{1}\right)\right)^{-1}\right) \\
& \equiv-\log \left(\Phi\left(\iota\left(\mathbf{x}_{0}\right), \iota\left(\mathbf{x}_{1}\right)\right) e^{-2 \pi i \iota\left(\mathbf{x}_{0}\right)}\right) \\
& \equiv 2 \pi i \iota\left(\mathbf{x}_{0}\right)-\sum_{k \geq 0} \frac{B_{k}}{k !} \operatorname{ad}^{k}(-\mathrm{b})\left(\varphi\left(\iota\left(\mathbf{x}_{0}\right), \iota\left(\mathbf{x}_{1}\right)\right)\right) \quad \bmod D^{2} \mathfrak{p}\left(E_{\tau}^{\times}\right) \mathbb{C} .
\end{aligned}
$$

Combining (5.3) and (5.4) and again applying [30], Corollary 3.24, we get

$$
\begin{aligned}
& \mathfrak{A}_{\infty} \equiv \mathfrak{T}+\sum_{n \geq 0} \frac{B_{n}}{n !} \operatorname{ad}^{n}(\mathfrak{T})(\mathfrak{S}) \\
& \equiv 2 \pi i \iota\left(\mathbf{x}_{0}\right)-\sum_{k \geq 0} \frac{B_{k}}{k !} \operatorname{ad}^{k}(-\mathrm{b})\left(\varphi\left(\iota\left(\mathbf{x}_{0}\right), \iota\left(\mathbf{x}_{1}\right)\right)\right)+\varphi\left(\iota\left(\mathbf{x}_{0}\right), \iota\left(\mathbf{x}_{1}\right)\right)+\pi i \iota\left(\mathbf{x}_{1}\right) \\
&+\sum_{n \geq 1} \frac{B_{n}}{n !} \operatorname{ad}^{n}\left(2 \pi i \iota\left(\mathbf{x}_{0}\right)\right)\left(\varphi\left(\iota\left(\mathbf{x}_{0}\right), \iota\left(\mathbf{x}_{1}\right)\right)+\pi i \iota\left(\mathbf{x}_{1}\right)\right) \\
& \equiv 2 \pi i \iota\left(\mathbf{x}_{0}\right)+\pi i \iota\left(\mathbf{x}_{1}\right)-\sum_{k \geq 1} \frac{B_{k}}{k !}\left((-1)^{k}-1\right) \operatorname{ad}^{k}(\mathrm{~b})\left(\varphi\left(\iota\left(\mathbf{x}_{0}\right), \iota\left(\mathbf{x}_{1}\right)\right)\right) \\
& \quad+\sum_{n \geq 1} \frac{B_{n}}{n !} \operatorname{ad}^{n}(\mathbf{b})\left(\pi i \iota\left(\mathbf{x}_{1}\right)\right)
\end{aligned}
$$




$$
\begin{aligned}
\equiv 2 \pi i \mathrm{~b} & +2 \pi i \sum_{k \geq 2} \frac{B_{k}}{k !} \mathrm{ad}^{k-1}(\mathrm{a})([\mathrm{a}, \mathrm{b}])-\operatorname{ad}(\mathrm{b})\left(\varphi\left(\iota\left(\mathrm{x}_{0}\right), \iota\left(\mathrm{x}_{1}\right)\right)\right) \\
& +\frac{2 \pi i}{2} \sum_{n \geq 1} \frac{B_{n}(2 \pi i)^{n}}{n !} \operatorname{ad}^{n}(\mathrm{~b})([\mathrm{a}, \mathrm{b}]) \bmod D^{2} \mathfrak{p}\left(E_{\tau}^{\times}\right) \mathbb{C}
\end{aligned}
$$

where in the last line, we have used that $B_{1}=-\frac{1}{2}$ and that $B_{2 n+1}=0$ for all $n \geq 1$. Using Lemma 5.1 together with Euler's formula $-\frac{\zeta(k)}{(-2 \pi i)^{k}}=\frac{B_{k}}{2 k !}$ for $k \geq 2$ even, it follows that (5.5) equals

$2 \pi i\left(\mathrm{~b}+\sum_{k \geq 2} \frac{B_{k}}{k !} \mathrm{ad}^{k-1}(\mathrm{a})([\mathrm{a}, \mathrm{b}])-\frac{2 \pi i}{4} \mathrm{ad}(\mathrm{b})([\mathrm{a}, \mathrm{b}])+\sum_{n \geq 3, \text { odd }} \zeta(n) \operatorname{ad}^{n}(\mathrm{~b})([\mathrm{a}, \mathrm{b}])\right)$.

Under the substitution $\operatorname{ad}^{k}(\mathrm{a}) \mathrm{ad}^{l}(\mathrm{~b})([\mathrm{a}, \mathrm{b}]) \mapsto \mathrm{U}^{k} \mathrm{~V}^{l}$ (cf. (3.2)), (5.1) now follows immediately from (5.6) (the $2 \pi i$ b-term belongs to $\mathfrak{A}_{\infty}^{(0)}$ and does not contribute to $\left.\mathfrak{A}_{\infty}^{(1)}\right)$. The calculation of $\mathfrak{B}_{\infty}^{(1)}$ is very similar, so we will omit some details. First, by definition

$$
\mathfrak{B}_{\infty}=\log \left(\Phi\left(\iota\left(\mathbf{x}_{\infty}\right), \iota\left(\mathbf{x}_{1}\right)\right) e^{\mathrm{a}} \Phi\left(\iota\left(\mathbf{x}_{0}\right), \iota\left(\mathbf{x}_{1}\right)\right)^{-1}\right),
$$

where $\mathbf{x}_{\infty}:=-\mathbf{x}_{0}-\mathbf{x}_{1}$. Furthermore,

$$
\begin{aligned}
\mathfrak{T} & :=\log \left(e^{\mathrm{a}} \Phi\left(\iota\left(\mathbf{x}_{0}\right), \iota\left(\mathbf{x}_{1}\right)\right)^{-1}\right) \\
& \equiv-\log \left(\Phi\left(\iota\left(\mathbf{x}_{0}\right), \iota\left(\mathbf{x}_{1}\right)\right) e^{-\mathrm{a}}\right) \\
& \equiv \mathrm{a}-\sum_{k \geq 0} \frac{B_{k}}{k !} \operatorname{ad}^{k}(-\mathbf{a})\left(\varphi\left(\iota\left(\mathbf{x}_{0}\right), \iota\left(\mathbf{x}_{1}\right)\right)\right) \bmod D^{2} \mathfrak{p}\left(E_{\tau}^{\times}\right) \mathbb{C} .
\end{aligned}
$$

We obtain

$$
\begin{aligned}
\mathfrak{B}_{\infty} & \equiv \log \left(\Phi\left(\iota\left(\mathbf{x}_{\infty}\right), \iota\left(\mathbf{x}_{1}\right)\right) e^{\mathfrak{T}}\right) \\
& \equiv \mathfrak{T}+\sum_{k \geq 0} \frac{B_{k}}{k !} \operatorname{ad}^{k}(\mathbf{a})\left(\varphi\left(\iota\left(\mathbf{x}_{\infty}\right), \iota\left(\mathbf{x}_{1}\right)\right)\right) \bmod D^{2} \mathfrak{p}\left(E_{\tau}^{\times}\right)_{\mathbb{C}}
\end{aligned}
$$

where the last equality follows from the fact that $\mathfrak{T} \equiv \mathrm{a} \bmod D^{1} \mathfrak{p}\left(E_{\tau}^{\times}\right) \mathbb{C} \cdot$ A short calculation shows that

$$
\begin{aligned}
& \mathfrak{T}+\sum_{k \geq 0} \frac{B_{k}}{k !} \operatorname{ad}^{k}(\mathrm{a})\left(\varphi\left(\iota\left(\mathbf{x}_{\infty}\right), \iota\left(\mathbf{x}_{1}\right)\right)\right) \\
& \equiv \mathrm{a}-\sum_{k \geq 0} \frac{B_{k}}{k !}(-1)^{k} \operatorname{ad}^{k}(\mathbf{a})\left(\varphi\left(\iota\left(\mathbf{x}_{0}\right), \iota\left(\mathbf{x}_{1}\right)\right)\right)+\sum_{k \geq 0} \frac{B_{k}}{k !} \operatorname{ad}^{k}(\mathbf{a})\left(\varphi\left(\iota\left(\mathbf{x}_{\infty}\right), \iota\left(\mathbf{x}_{1}\right)\right)\right)
\end{aligned}
$$




$$
\equiv \mathrm{a}+\sum_{k \geq 0} \frac{B_{k}}{k !} \operatorname{ad}^{k}(\mathbf{a})\left(\varphi\left(\iota\left(\mathbf{x}_{\infty}\right), \iota\left(\mathbf{x}_{1}\right)\right)-(-1)^{k} \varphi\left(\iota\left(\mathbf{x}_{0}\right), \iota\left(\mathbf{x}_{1}\right)\right)\right) \bmod D^{2} \mathfrak{p}\left(E_{\tau}^{\times}\right) \mathbb{C}
$$

The term in brackets is equal to

$$
\begin{cases}2 \sum_{n \geq 2, \text { even }} \zeta(n) \mathrm{ad}^{n-1}(\mathrm{~b})([\mathrm{a}, \mathrm{b}]) & \text { if } k \text { is even } \\ -2 \sum_{n \geq 3 \text { odd }} \zeta(n) \mathrm{ad}^{n-1}(\mathrm{~b})([\mathrm{a}, \mathrm{b}]) & \text { if } k \text { is odd }\end{cases}
$$

Again using that $\zeta(k)=-\frac{B_{k}(2 \pi i)^{k}}{2 k !}$, if $k \geq 2$ is even, we obtain that (5.7) equals

$$
\begin{aligned}
& \mathrm{a}-\sum_{n \geq 2} \frac{B_{n}(2 \pi i)^{n}}{n !} \operatorname{ad}^{n-1}(\mathrm{~b})([\mathrm{a}, \mathrm{b}])-\sum_{k, n \geq 2} \frac{B_{k} B_{n}(2 \pi i)^{n}}{k ! n !} \operatorname{ad}^{k}(\mathrm{a}) \operatorname{ad}^{n-1}(\mathrm{~b})([\mathrm{a}, \mathrm{b}]) \\
& \sum_{+n \geq 3, \text { odd }} \zeta(n) \operatorname{ad}(\mathrm{a}) \operatorname{ad}^{n-1}(\mathrm{~b})([\mathrm{a}, \mathrm{b}]) \\
& \bmod D^{2} \mathfrak{p}\left(E_{\tau}^{\times}\right) \mathbb{C}
\end{aligned}
$$

The first term a belongs to $\mathfrak{B}_{\infty}^{(0)}$, and does not contribute to $\mathfrak{B}_{\infty}^{(1)}$. Applying the isomorphism (3.2) to the remaining terms in (5.8), we obtain the desired result (5.2).

The series $\mathfrak{A}_{\infty}^{(1)}$ and $\mathfrak{B}_{\infty}^{(1)}$ are closely related to the extended period polynomials of Eisenstein series $r_{G_{2 k}}(\mathrm{X}, \mathrm{Y})$ [33]. Precisely, for $k \geq 2$, one has

$$
r_{G_{2 k}}(\mathrm{X}, \mathrm{Y})=\omega_{G_{2 k}}^{+} P_{G_{2 k}}(\mathrm{X}, \mathrm{Y})^{+}+\omega_{G_{2 k}}^{-} P_{G_{2 k}}(\mathrm{X}, \mathrm{Y})^{-}
$$

where

$$
\begin{aligned}
& P_{G_{2 k}}(\mathrm{X}, \mathrm{Y})^{+}=\mathrm{X}^{2 k-2}-\mathrm{Y}^{2 k-2} \\
& P_{G_{2 k}}(\mathrm{X}, \mathrm{Y})^{-}=\sum_{-1 \leq n \leq 2 k-1} \frac{B_{n+1} B_{2 k-n-1}}{(n+1) !(2 k-1-n) !} \mathrm{X}^{n} \mathrm{Y}^{2 k-2-n}
\end{aligned}
$$

and $\omega_{G_{2 k}}^{-}=-\frac{(2 k-2) !}{2}, \omega_{G_{2 k}}^{+}=\frac{\zeta(2 k-1)}{(2 \pi i)^{2 k-1}} \omega_{G_{2 k}}^{-}$(the "periods" of $\left.G_{2 k}\right)$. Now let

$$
\widetilde{\mathfrak{A}}(\mathrm{U}, \mathrm{V})=\frac{1}{\mathrm{~V}} \mathfrak{A}_{\infty}^{(1)}(\mathrm{U}, \mathrm{V}), \quad \widetilde{\mathfrak{B}}(\mathrm{U}, \mathrm{V})=\frac{1}{\mathrm{U}} \mathfrak{B}_{\infty}^{(1)}(\mathrm{U}, \mathrm{V})
$$

These are formal Laurent series in the variables $\mathrm{U}$ and $\mathrm{V}$. In general, if $f(\mathrm{U}, \mathrm{V})$ is a formal Laurent series, we denote by $f(\mathrm{U}, \mathrm{V})_{k}$ its homogeneous component of degree $k$ and $f(\mathrm{U}, \mathrm{V})^{ \pm}:=\frac{f(\mathrm{U}, \mathrm{V}) \pm f(-\mathrm{U}, \mathrm{V})}{2}$. Comparing now (5.9) with Theorem 5.2, we get 
Corollary 5.3 We have

$r_{G_{2 k}}(\mathrm{U}, \mathrm{V})=\frac{\omega_{G_{2 k}}^{-}}{2 \pi i}\left[\tilde{\mathfrak{A}}(\overline{\mathrm{U}}, \mathrm{V})_{2 k-2}^{+}+\widetilde{\mathfrak{B}}(\mathrm{V}, \overline{\mathrm{U}})_{2 k-2}^{+}-\widetilde{\mathfrak{A}}(\overline{\mathrm{U}}, \mathrm{V})_{2 k-2}^{-}-\widetilde{\mathfrak{B}}(\overline{\mathrm{U}}, \mathrm{V})_{2 k-2}^{-}\right]$ where $\overline{\mathrm{U}}=\frac{\mathrm{U}}{2 \pi i}$.

\subsection{The geometric piece: special values of elliptic polylogarithms}

Recall from Sect. 4.2 the definition of the automorphism $g(\tau): \exp \mathfrak{p}\left(E_{\tau}^{\times}\right) \mathbb{C} \rightarrow$ $\exp \mathfrak{p}\left(E_{\tau}^{\times}\right) \mathbb{C}$. It naturally extends to the topological enveloping algebra $\mathbb{Q}\langle\langle\mathrm{a}, \mathrm{b}\rangle\rangle$ of $\mathfrak{p}\left(E_{\tau}^{\times}\right) \mathbb{C}$.

In this section, we compute the images of $g(\tau)(\mathrm{a}), g(\tau)(\mathrm{b})$ in the meta-abelian quotient $\mathfrak{p}\left(E_{\tau}^{\times}\right)_{\mathbb{C}}^{\text {met-ab }}$ of $\mathfrak{p}\left(E_{\tau}^{\times}\right)_{\mathbb{C}}$, and relate the result to special values of BeilinsonLevin's elliptic polylogarithms [1,22].

Theorem 5.4 Let $\mathrm{W}=\frac{\mathrm{U}}{2 \pi i}+\tau \mathrm{V}$. We have

$$
g(\tau)(\mathrm{a})^{\mathrm{met}-\mathrm{ab}}=\mathrm{a}+2 \pi i \tau \mathrm{b}-2 \pi i \mathrm{~W} \sum_{k=1}^{\infty} \frac{2}{(2 k-2) !} \int_{\tau}^{\overrightarrow{1}_{\infty}} \underline{G}_{2 k}
$$

and

$$
g(\tau)(\mathrm{b})^{\mathrm{met}-\mathrm{ab}}=2 \pi i \mathrm{~b}-2 \pi i \mathrm{~V} \sum_{k=1}^{\infty} \frac{2}{(2 k-2) !} \int_{\tau}^{\overrightarrow{1}_{\infty}} \underline{G}_{2 k},
$$

where $\underline{G}_{2 k}=(2 \pi i)^{2 k-1} G_{2 k}(z)(\mathrm{W}-z \mathrm{~V})^{2 k-2} \mathrm{~d} z$.

Proof By Corollary 4.4, we have

$$
\begin{aligned}
g(\tau)(\mathrm{a})^{\mathrm{met}-\mathrm{ab}}= & \mathrm{a}+2 \pi i \tau \mathrm{b}+\sum_{n \geq 0, k \geq 1}(-2 \pi i)^{n+1} \mathcal{G}\left(\{0\}_{n}, 2 k ; \tau\right)\left(\varepsilon_{0}^{n} \circ \varepsilon_{2 k}\right)(\mathrm{a}) \\
& +\sum_{k, n \geq 1}(-2 \pi i)^{n+1} \mathcal{G}\left(\{0\}_{n-1}, 2 k, 0 ; \tau\right)\left(\varepsilon_{0}^{n-1} \circ \varepsilon_{2 k} \circ \varepsilon_{0}\right)(\mathrm{a}) \\
= & \mathrm{a}+2 \pi i \tau \mathrm{b}+\sum_{n \geq 0, k \geq 1} \frac{2(-2 \pi i)^{n+1}}{(2 k-2) !} \mathcal{G}\left(\{0\}_{n}, 2 k ; \tau\right) \varepsilon_{0}^{n}\left(\mathrm{ad}^{2 k-1}(\mathrm{a})([\mathrm{a}, \mathrm{b}])\right) \\
& -\sum_{k, n \geq 1} \frac{2(-2 \pi i)^{n+1}}{(2 k-2) !} \mathcal{G}\left(\{0\}_{n-1}, 2 k, 0 ; \tau\right) \varepsilon_{0}^{n-1}\left(\operatorname{ad}^{2 k-2}(\mathrm{a}) \operatorname{ad}(\mathrm{b})([\mathrm{a}, \mathrm{b}])\right),
\end{aligned}
$$

Using the isomorphism of Proposition 3.2 together with Proposition 3.4 and Proposition 2.3, we see that (5.12) equals

$$
\mathrm{a}+2 \pi i \tau \mathrm{b}-\sum_{n \geq 0, k \geq 1} \frac{2(2 \pi i)^{n+1}}{(2 k-2) ! n !} I_{n}\left(G_{2 k} ; \tau\right)\left(\mathrm{V} \frac{\partial}{\partial \mathrm{U}}\right)^{n} \mathrm{U}^{2 k-1}
$$




$$
-\sum_{k, n \geq 1} \frac{2(2 \pi i)^{n+1}}{(2 k-2) !(n-1) !}\left(\tau I_{n-1}\left(G_{2 k} ; \tau\right)-I_{n}\left(G_{2 k} ; \tau\right)\right)\left(\mathrm{V} \frac{\partial}{\partial \mathrm{U}}\right)^{n-1} \mathrm{U}^{2 k-2} \mathrm{~V}
$$

Now we apply the differential operator $\mathrm{V} \frac{\partial}{\partial U}$ and split the first and the last sum to obtain

$$
\begin{aligned}
g(\tau)(\mathrm{a})^{\mathrm{met}-\mathrm{ab}}= & \mathrm{a}+2 \pi i \tau \mathrm{b}-\sum_{k \geq 1} \frac{2(2 \pi i)}{(2 k-2) !} I_{0}\left(G_{2 k} ; \tau\right) \mathrm{U}^{2 k-1} \\
& -\sum_{k, n \geq 1} \frac{2(2 k-1)(2 \pi i)^{n+1}}{(2 k-1-n) ! n !} I_{n}\left(G_{2 k} ; \tau\right) \mathrm{U}^{2 k-1-n} \mathrm{~V}^{n} \\
& -2 \pi i \tau \sum_{k, n \geq 1} \frac{2(2 \pi i)^{n}}{(2 k-1-n) !(n-1) !} I_{n-1}\left(G_{2 k} ; \tau\right) \mathrm{U}^{2 k-1-n} \mathrm{~V}^{n-1} \\
& +\sum_{k, n \geq 1} \frac{2(2 \pi i)^{n+1}}{(2 k-1-n) !(n-1) !} I_{n}\left(G_{2 k} ; \tau\right) \mathrm{U}^{2 k-1-n} \mathrm{~V}^{n-1} .
\end{aligned}
$$

From the definition of $I_{n}\left(G_{2 k} ; \tau\right)$, it is easy to see that the third sum equals

$$
-2 \pi i \tau \mathrm{V} \sum_{k=1}^{\infty} \frac{2(2 \pi i)}{(2 k-2) !} \int_{\tau}^{\overrightarrow{1}_{\infty}} G_{2 k}(z)(\mathrm{U}+2 \pi i(\tau-z) \mathrm{V})^{2 k-2} \mathrm{~d} z
$$

On the other hand, the first, second and fourth sum give

$$
-\mathrm{U} \sum_{k=1}^{\infty} \frac{2(2 \pi i)}{(2 k-2) !} \int_{\tau}^{\overrightarrow{1}_{\infty}} G_{2 k}(z)(\mathrm{U}+2 \pi i(\tau-z) \mathrm{V})^{2 k-2} \mathrm{~d} z
$$

Combining the two equations and setting $\mathrm{W}=\frac{U}{2 \pi i}+\tau \mathrm{V}$, the first equality (5.10) follows. Since $g(\tau)$ is uniquely determined by its value on $e^{\mathrm{a}}$, the second statement (5.11) follows from the first, but can also be proved directly along similar lines.

We now give the relation to special values of elliptic polylogarithms. Following the notation of [22], we let $\Xi(\xi, \tau ; \mathrm{X}, \mathrm{Y})$ be the (modified) generating series of elliptic polylogarithms $\Lambda_{m, n}(\xi, \tau)$. These are holomorphic functions on the universal covering of the once-punctured elliptic curve $E_{\tau}^{\times}$, which are obtained by averaging the (Debye) polylogarithms along the spiral $q^{\mathbb{Z}}$. Let

$$
\Xi^{*}(0, \tau ; \mathrm{X}, \mathrm{Y}):=\left.\left(\Xi(\xi, \tau ; \mathrm{X}, \mathrm{Y})-\frac{1}{2 \pi i} \log (2 \pi i \xi)\right)\right|_{\xi=0}
$$


be its (regularized) special value at the zero section of the elliptic curve. It has been shown in [22], Theorem 4.1 that

$$
\Xi^{*}(0, \tau ; X, Y)=\frac{-\tau}{X(X-\tau Y)}+\sum_{k=2}^{\infty}(-1)^{k-1}(k-1) \mathcal{E}_{k}
$$

where for $k \geq 2, \mathcal{E}_{k}$ is the indefinite integral of $E_{k}(\tau)(X-\tau Y)^{k-2} \mathrm{~d} \tau$ with $E_{k}(\tau)=$ $\frac{2(2 \pi i)^{k}}{(k-1) !} G_{k}(\tau)=\sum_{(m, n) \in \mathbb{Z}^{2} \backslash\{(0,0)\}} \frac{1}{(m \tau+n)^{k}}$ the classical Eisenstein series of weight $k$. The constants of integration in the indefinite integrals can be retrieved uniformly as the (regularized) special value of $\Xi^{*}(0, \tau ; X, Y)$ at $\tau=i \infty$, which is straightforwardly computed from the definitions and is given explicitly by

$$
\Xi^{*}(0, i \infty ; X, Y)=-\sum_{n \geq 2} \frac{\zeta(n)}{(2 \pi i)^{n}} Y^{n-1}+\frac{1}{e^{X}-1}\left(\frac{1}{e^{Y}-1}-\frac{1}{Y}\right) .
$$

Now comparing (5.13) with Theorem 5.4, we obtain

Corollary 5.5 Let $g(\tau)(a)^{\text {met-ab }}-a$, and replace $2 \pi i$ b by $(\mathrm{W}-\tau \mathrm{V})^{-1}$. Then

$$
\frac{g(\tau)(\mathrm{a})^{\mathrm{met}-\mathrm{ab}}-\mathrm{a}}{-(2 \pi i)^{2} \mathrm{~W}}=\Xi^{*}(0, \tau ; 2 \pi i \mathrm{~W}, 2 \pi i \mathrm{~V})-\Xi^{*}(0, i \infty ; 2 \pi i \mathrm{~W}, 2 \pi i \mathrm{~V}),
$$

where $\Xi^{*}(0, i \infty ; X, Y)$ is given in (5.14) above.

\subsection{Putting the pieces together}

We can now complete the computation of $\mathfrak{A}(\tau)^{\text {met-ab }}$ and $\mathfrak{B}(\tau)^{\text {met-ab }}$ by combining the results of the previous sections.

Theorem 5.6 We have

$$
\mathfrak{A}(\tau)^{\mathrm{met}-\mathrm{ab}}=2 \pi i \mathrm{~b}+\exp \left(\tau \frac{\partial}{\partial \overline{\mathrm{U}}} \mathrm{V}\right) \mathfrak{A}_{\infty}^{(1)}-2 \pi i \mathrm{~V} \sum_{k=1}^{\infty} \frac{2}{(2 k-2) !} \int_{\tau}^{\overrightarrow{1}_{\infty}} \underline{G}_{2 k}
$$

and

$$
\mathfrak{B}(\tau)^{\mathrm{met}-\mathrm{ab}}=\mathrm{a}+2 \pi i \tau \mathrm{b}+\exp \left(\tau \frac{\partial}{\partial \overline{\mathrm{U}}} \mathrm{V}\right) \mathfrak{B}_{\infty}^{(1)}-2 \pi i \mathrm{~W} \sum_{k=1}^{\infty} \frac{2}{(2 k-2) !} \int_{\tau}^{\overrightarrow{1}_{\infty}} \underline{G}_{2 k}
$$

where $\overline{\mathrm{U}}=\frac{\mathrm{U}}{2 \pi i}, \mathrm{~W}=\overline{\mathrm{U}}+\tau \mathrm{V}$ and $\mathfrak{A}_{\infty}^{(1)}$ and $\mathfrak{B}_{\infty}^{(1)}$ are as given in Theorem 5.2

Proof We only prove the first equality, the second one is shown analogously. By Theorem 4.3, we have $\mathfrak{A}(\tau)=g(\tau)\left(\mathfrak{A}_{\infty}\right)$, hence

$$
\mathfrak{A}(\tau)^{\mathrm{met}-\mathrm{ab}} \equiv g(\tau)\left(\mathfrak{A}_{\infty}\right) \quad \bmod D^{2} \mathfrak{p}\left(E_{\tau}^{\times}\right) \mathbb{C},
$$


and from Proposition 3.4, we get

$$
\mathfrak{A}(\tau)^{\mathrm{met}-\mathrm{ab}}=g(\tau)\left(\mathfrak{A}_{\infty}^{(1)}\right)+2 \pi i g(\tau)(\mathrm{b})^{\mathrm{met}-\mathrm{ab}} .
$$

The only derivation which acts non-trivially on $\operatorname{gr}_{D}^{1} \mathfrak{p}\left(E_{\tau}^{\times}\right) \mathbb{C}$ is $\varepsilon_{0}$ which itself acts as $-\frac{\partial}{\partial U} \mathrm{~V}=\frac{1}{2 \pi i} \frac{\partial}{\partial \overline{\mathrm{U}}} \mathrm{V}$. Combining this with Theorem 5.4, we get the result:

$$
\mathfrak{A}(\tau)^{\mathrm{met}-\mathrm{ab}}=2 \pi i \mathrm{~b}+\exp \left(\tau \frac{\partial}{\partial \overline{\mathrm{U}}} \mathrm{V}\right) \mathfrak{A}_{\infty}^{(1)}-2 \pi i \mathrm{~V} \sum_{k=1}^{\infty} \frac{2}{(2 k-2) !} \int_{\tau}^{\overrightarrow{1}_{\infty}} \underline{G}_{2 k}
$$

Remark 5.7 The value for $\mathfrak{A}(\tau)^{\text {met-ab }}$ given in Theorem 5.6 can be further simplified. To this end, recall from Theorem 5.2 that

$$
\mathfrak{A}_{\infty}^{(1)}=2 \pi i\left(\sum_{k=1}^{\infty} \frac{B_{2 k}}{(2 k) !} \mathrm{U}^{2 k-1}-\frac{2 \pi i}{4} \mathrm{~V}+\sum_{n=3, \text { odd }} \zeta(n) \mathrm{V}^{n}\right)
$$

Therefore

$$
\begin{aligned}
\exp \left(\tau \frac{\partial}{\partial \overline{\mathrm{U}}} \mathrm{V}\right) \mathfrak{A}_{\infty}^{(1)} & =\mathfrak{A}_{\infty}^{(1)}+2 \pi i \sum_{k, n \geq 1} \frac{\tau^{n}}{n !} \frac{B_{2 k}}{(2 k) !}\left(\frac{\partial}{\partial \overline{\mathrm{U}}} \mathrm{V}\right)^{n} \mathrm{U}^{2 k-1} \\
& =\mathfrak{A}_{\infty}^{(1)}+2 \pi i \mathrm{~V} \sum_{k, n \geq 1} \frac{2(2 \pi i)^{2 k-1}}{(2 k-2) !}\left[\frac{\tau^{n}}{n !} \frac{B_{2 k}}{4 k}\left(\frac{\partial}{\partial \overline{\mathrm{U}}} \mathrm{V}\right)^{n-1} \overline{\mathrm{U}}^{2 k-2}\right] \\
& =\mathfrak{A}_{\infty}^{(1)}+2 \pi i V \sum_{k, n \geq 1} \frac{2(2 \pi i)^{2 k-1}}{(2 k-1-n) !}\left[\frac{\tau^{n}}{n !} \frac{B_{2 k}}{4 k} \overline{\mathrm{U}}^{2 k-1-n} \mathrm{~V}^{n-1}\right] \\
& =\mathfrak{A}_{\infty}^{(1)}+2 \pi i \mathrm{~V} \sum_{k=1}^{\infty} \frac{2(2 \pi i)^{2 k-1}}{(2 k-2) !} \frac{B_{2 k}}{4 k} \int_{0}^{\tau}(\overline{\mathrm{U}}+(\tau-z) \mathrm{V})^{2 k-2} \mathrm{~d} z
\end{aligned}
$$

Note that $-\frac{B_{2 k}}{4 k}=a_{0}\left(G_{2 k}\right)$, the zeroth Fourier coefficient of $G_{2 k}$. Consequently, we obtain

$$
\mathfrak{A}(\tau)^{\mathrm{met}-\mathrm{ab}}=2 \pi i \mathrm{~b}+\mathfrak{A}_{\infty}^{(1)}-2 \pi i \mathrm{~V} \sum_{k=1}^{\infty} \frac{2}{(2 k-2) !} \int_{\tau}^{i \infty} \underline{G}_{2 k}^{0}
$$

where $\underline{G}_{2 k}^{0}=\underline{G}_{2 k}-a_{0}\left(\underline{G}_{2 k}\right)=\underline{G}_{2 k}-(2 \pi i)^{2 k-1} a_{0}\left(G_{2 k}\right)(\mathrm{W}-z \mathrm{~V})^{2 k-2}$, since

$$
\int_{\tau}^{\overrightarrow{1}_{\infty}} \underline{G}_{2 k}=\int_{\tau}^{i \infty} \underline{G}_{2 k}^{0}-\int_{0}^{\tau} a_{0}\left(\underline{G}_{2 k}\right)
$$


Acknowledgements Open access funding provided by Max Planck Society. Very many thanks to B. Enriquez and H. Nakamura for very inspiring discussions at the conference "GRT, MZVs and associators" in Les Diablerets in 2015, which formed the starting point of this project. Thanks are also due to A. Alekseev for the invitation to that conference. Also, many thanks to F. Brown, B. Enriquez, H. Furusho and F. Zerbini for helpful comments on an earlier version of this paper. This paper was written while the author was a Ph.D. student at Universität Hamburg under the supervision of U. Kühn.

Open Access This article is distributed under the terms of the Creative Commons Attribution 4.0 International License (http://creativecommons.org/licenses/by/4.0/), which permits unrestricted use, distribution, and reproduction in any medium, provided you give appropriate credit to the original author(s) and the source, provide a link to the Creative Commons license, and indicate if changes were made.

\section{References}

1. Beřlinson, A., Levin, A.: The elliptic polylogarithm. In: Motives (Seattle, WA, 1991), Proc. Sympos. Pure Math., vol. 55, pp. 123-190. American Mathematical Society, Providence, RI (1994)

2. Broedel, J., Matthes, N., Schlotterer, O.: Relations between elliptic multiple zeta values and a special derivation algebra. J. Phys. A 49(15), 155-203 (2016)

3. Brown, F.: Mixed Tate motives over $\mathbb{Z}$. Ann. Math. (2) 175(2), 949-976 (2012)

4. Brown, F.: On the decomposition of motivic multiple zeta values. In: Galois-Teichmüller theory and arithmetic geometry. Advanced Studies in Pure Math, vol. 63, pp. 31-58. Mathematical Society of Japan, Tokyo (2012)

5. Brown, F.: Depth-graded motivic multiple zeta values. arXiv:1301.3053 (2013)

6. Brown, F.: Multiple modular values and the relative completion of the fundamental group of $\mathcal{M}_{1,1}$. arXiv:1407.5167v3 (2016)

7. Brown, F.: Zeta elements in depth 3 and the fundamental Lie algebra of the infinitesimal Tate curve. Forum Math. Sigma 5, e1, 56. https://doi.org/10.1017/fms.2016.29 (2017)

8. Brown, F., Levin, A.: Multiple elliptic polylogarithms. arXiv:1110.6917 (2011)

9. Calaque, D., Enriquez, B., Etingof, P.: Universal KZB equations: the elliptic case. In: Algebra, arithmetic, and geometry: in honor of Yu. I. Manin. Vol. I, Progr. Math., vol. 269, pp. 165-266. Birkhäuser Boston, Inc., Boston, MA (2009)

10. Chen, K.T.: Iterated path integrals. Bull. Am. Math. Soc. 83(5), 831-879 (1977)

11. Deligne, P.: Le groupe fondamental de la droite projective moins trois points. In: Galois groups over Q (Berkeley, CA, 1987), Math. Sci. Res. Inst. Publ., vol. 16, pp. 79-297. Springer, New York (1989)

12. Deligne, P., Goncharov, A.B.: Groupes fondamentaux motiviques de Tate mixte. Ann. Sci. École Norm. Supér. (4) 38(1), 1-56 (2005)

13. Drinfel'd, V.G.: On quasitriangular quasi-Hopf algebras and on a group that is closely connected with $\mathrm{Gal}(\overline{\mathbf{Q}} / \mathbf{Q})$. Algebra i Analiz 2(4), 149-181 (1990)

14. Enriquez, B.: Elliptic associators. Sel. Math. (N.S.) 20(2), 491-584 (2014)

15. Enriquez, B.: Analogues elliptiques des nombres multizétas. Bull. Soc. Math. Fr. 144(3), 395-427 (2016)

16. Goncharov, A.B.: Multiple polylogarithms and mixed Tate motives. arXiv:math/0103059 (2001)

17. Hain, R.: Notes on the Universal Elliptic KZB Equation. arXiv:1309.0580 (2013)

18. Hain, R.: The Hodge-de Rham theory of modular groups. In: Recent Advances in Hodge Theory, London Math. Soc. Lecture Note Ser., vol. 427, pp. 422-514. Cambridge University Press, Cambridge (2016)

19. Hain, R., Matsumoto, M.: Universal Mixed Elliptic Motives. arXiv:1512.03975 (2015)

20. Ihara, K., Kaneko, M., Zagier, D.: Derivation and double shuffle relations for multiple zeta values. Compos. Math. 142(2), 307-338 (2006)

21. Ihara, Y.: Profinite braid groups, Galois representations and complex multiplications. Ann. Math. (2) 123(1), 43-106 (1986)

22. Levin, A.: Elliptic polylogarithms: an analytic theory. Compos. Math. 106(3), 267-282 (1997)

23. A. Levin and G. Racinet. Towards multiple elliptic polylogarithms. arXiv:math/0703237 2007

24. Lochak, P., Matthes, N., Schneps, L.: Elliptic multiple zeta values and the elliptic double shuffle relations. arXiv:1703.09410 (2017) 
25. Manin, Y.I.: Iterated integrals of modular forms and noncommutative modular symbols. In: Ginzburg, V. (ed.) Algebraic geometry and number theory, Progr. Math., vol. 253, pp. 565-597. Birkhäuser Boston, Boston, MA (2006)

26. Matthes, N.: Elliptic multiple zeta values. Ph.D. thesis, Universität Hamburg (2016)

27. Matthes, N.: Elliptic double zeta values. J. Number Theory 171, 227-251 (2017)

28. Nakamura, H.: On exterior Galois representations associated with open elliptic curves. J. Math. Sci. Univ. Tokyo 2(1), 197-231 (1995)

29. Nakamura, H.: On profinite Eisenstein periods in the monodromy of universal elliptic curves. http:// www.math.sci.osaka-u.ac.jp/ nakamura/zoo/fox/EisenRevisited.pdf (2016)

30. Reutenauer, C.: Free Lie algebras, volume 7 of London Mathematical Society Monographs. New Series. The Clarendon Press, Oxford University Press, New York (1993). Oxford Science Publications

31. Serre, J.-P.: Lie algebras and Lie groups, Lecture Notes in Mathematics, vol 1500. Springer, Berlin (2006). 1964 lectures given at Harvard University, Corrected fifth printing of the second (1992) edition

32. Tsunogai, H.: On some derivations of Lie algebras related to Galois representations. Publ. Res. Inst. Math. Sci. 31(1), 113-134 (1995)

33. Zagier, D.: Periods of modular forms and Jacobi theta functions. Invent. Math. 104(3), 449-465 (1991)

34. Zagier, D.: Periods of modular forms, traces of Hecke operators, and multiple zeta values. Sūrikaisekikenkyūsho Kōkyūroku, (843):162-170 (1993). Research into automorphic forms and $L$ functions (Japanese) (Kyoto, 1992) 